\title{
Risk factors for delayed isolation of tuberculosis patients in a tertiary care hospital in a low-incidence country
}

\author{
J Severin", S Ruhe-van der Werff, M Bakker, MC Vos \\ From 3rd International Conference on Prevention and Infection Control (ICPIC 2015) \\ Geneva, Switzerland. 16-19 June 2015
}

\section{Introduction}

Delayed isolation of tuberculosis patients increases the risk of nosocomial transmission.

\section{Objectives}

The purpose of this study was to analyze risk factors for delayed isolation of tuberculosis patients in a tertiary care hospital in the Netherlands.

\section{Methods}

We included all patients with culture-proven Mycobacterium tuberculosis infection between January $1^{\text {st }} 2010$ and December $31^{\text {st }}$ 2012. Demographic and clinical information was collected retrospectively from the medical records. Risk factors were analyzed using univariate and multivariate logistic regression. A prediction model for non-isolation was developed.

\section{Results}

In the three-year time period, 58 patients had a cultureproven tuberculosis infection in our hospital, 20 with an extrapulmonary infection only. For 41 patients (70.7\%), isolation measures were not taken. Twelve of these were patients with extrapulmonary tuberculosis only, and in these cases isolation measures were forgotten with aerosol generating procedures. The median number of days of non-isolation was 4 (range 1-162). For the patients with pulmonary tuberculosis (38 patients, 25 (65.8\%) not isolated) four factors were predictive for non-isolation: presence of the symptom "fatigue", absence of hemoptysis, absence of tuberculosis in the differential diagnosis, absence of involvement of a pulmonologist or infectious diseases specialist on admission. Due to these non-isolated

Erasmus MC, Rotterdam, Netherlands 